\title{
Simulation of Normal Incidence Sound Absorption Coefficients of Perforated Panels With/Without Glass Wool by Transmission Line Parameters in a Two-Port Network
}

\author{
Takayoshi Nakai* \\ Department of Electric and Electronic Engineering, Shizuoka University, \\ Hamamatsu-shi, Japan. \\ *Corresponding author: nakai.takayoshi@shizuoka.ac.jp
}

\begin{abstract}
This paper describes simulation of normal incidence sound absorption coefficients of perforated panels by transmission line parameters in a two-port network. Maa and Sakagami have investigated micro perforated panels, MPP. But their theories can treat only near $1 \%$ perforation rates of perforated panels with back cavities. If sound propagates as a plane wave, sound propagation can be represented as transmission line parameters in a two-port network. Perforated panels, back cavities, and glass wool absorption materials are represented as matrix of transmission line parameters, respectively. Transmission line parameters of a perforated panel with a back cavity are calculated as multiplication of their matrices. An input impedance can be calculated from the transmission line parameters. A normal incident absorption coefficient is calculated from the input impedance. Holes of the perforated panels have losses of viscous friction and thermal conduction at their walls. Simulations are done in the condition of $0.25 \mathrm{~mm}$ to $5 \mathrm{~mm}$ diameters of holes, $0.25 \%$ to $25 \%$ perforation rates, $0.5 \mathrm{~mm}$ to $5 \mathrm{~mm}$ thickness of the perforated panels with back cavities in which there are or are not glass wool absorption materials. The results of these simulations are good agreements with the results of our measurements by transfer function method except in the condition of more than $1 \mathrm{~mm}$ diameter of holes.
\end{abstract}

Keywords: Sound Absorption Coefficients; Perforated Panel; Glass Wool; Two Port Network;

\section{INTRODUCTION}

Maa and Sakagami have investigated micro perforated panels, MPP[1],[2]. But their theories can treat only near $1 \%$ perforation rates of perforated panels with back cavities. Our aim is development of an adequate theory in various conditions such as back cavities with/without glass wool, diameter of the holes on the panel and thickness of the panel. If sound propagates as a plane wave, sound propagation can be represented as transmission line parameters in a two-port network of distributed parameter circuit. In the next section, transmission line parameters are described. In the third section, simulations using transmission line parameters are described. In the fourth section, results and discussions are described.

\section{THEORY}

Analogies Between Acoustic and Electric Quantities

Sound generates due to time variations of pressure and volume velocity. When relationship between pressure and volume velocity is represented by physical equations, it can be represented by similar equations as relationship between voltage and current in 
electric circuits. Therefore, if sound propagates as a plane wave, sound propagation can be represented as transmission line parameters in a two-port network of distributed parameter circuit. Electric circuits analyzed acoustics are called as "acoustic equivalent circuits.”

For a lossless tube, the partial differential equations are given as follows [3].

$$
-\frac{\partial p}{\partial x}=\frac{p}{A} \frac{\partial u}{\partial t}, \quad-\frac{\partial u}{\partial x}=\frac{A}{p c^{2}} \frac{\partial u}{\partial t}
$$

where $x$ is the location in the tube, $t$ is the time, $\rho$ is the density of air, $c$ is the velocity in air, and $A$ is cross-sectional area of the tube. TABLE 1 shows analogies between acoustics and electric quantities.

TABLE 1. ANALOGIES BETWEEN ACOUSTICS AND ELECTRIC QUANTITIES.

\begin{tabular}{|c|c|}
\hline Acoustic Quantity & Analogous Electric Quantity \\
\hline$p:$ pressure & $v$ : voltage \\
\hline$u$ : volume velocity & $i:$ current \\
\hline$\rho / A:$ acoustic inductance & $L:$ inductance \\
\hline$A /\left(\rho c^{2}\right):$ acoustic capacitance & $C:$ capacitance \\
\hline
\end{tabular}

\section{Transmission Line Parameters in a Two-Port Network of Distributed Parameter} Circuit[4]

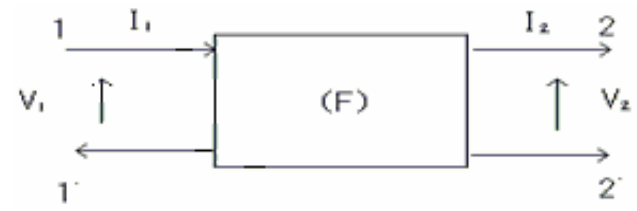

Fig. 1. Transmission line parameters in a two-port network

In a two-port network of distributed parameter circuit, there are various parameters, but in this paper transmission line parameters are used. Fig. 1 shows representation of transmission line parameters in a two-port network for the acoustic tube. As shown in Fig $1, \mathrm{~V}_{1}[\mathrm{~V}]$ and $\mathrm{I}_{1}[\mathrm{~A}]$ are voltage and current of input, and $\mathrm{V}_{2}[\mathrm{~V}]$ and $\mathrm{I}_{2}[\mathrm{~A}]$ are voltage and current of output. They are related as follows, and transmission line parameters matrix, $\mathrm{F}$, is defined as follows.

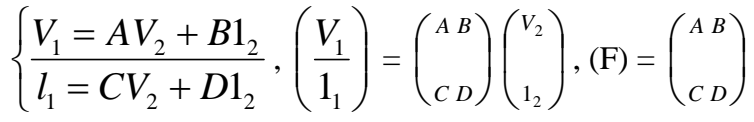

Units of transmission line parameters are $A[-], B[\Omega], C[S]$, and $D[-]$, respectively, and [-] means non-dimensional. Two transmission line parameters are given as follows. 


$$
\left(\begin{array}{c}
V_{2} \\
I_{1}
\end{array}\right)=\left(F_{1}\right)\left(\begin{array}{l}
V_{2} \\
I_{2}
\end{array}\right)=\left(\begin{array}{ll}
A_{1} & B_{1} \\
C_{1} & D_{1}
\end{array}\right)\left(\left(\begin{array}{c}
V_{2} \\
1_{2}
\end{array}\right), \quad\left(\begin{array}{c}
V_{2} \\
I_{2}
\end{array}\right)=\left(F_{2}\right)\left(\begin{array}{l}
V_{7} \\
I_{3}
\end{array}\right)=\left(\begin{array}{ll}
A_{2} & B_{2} \\
C_{2} & D_{2}
\end{array}\right)\left(\left(\begin{array}{c}
v_{3} \\
1_{3}
\end{array}\right)\right.\right.
$$

Then, the cascade connection of two transmission line parameters as shown in Fig. 2, is given as multiplication of matrixes as follows.

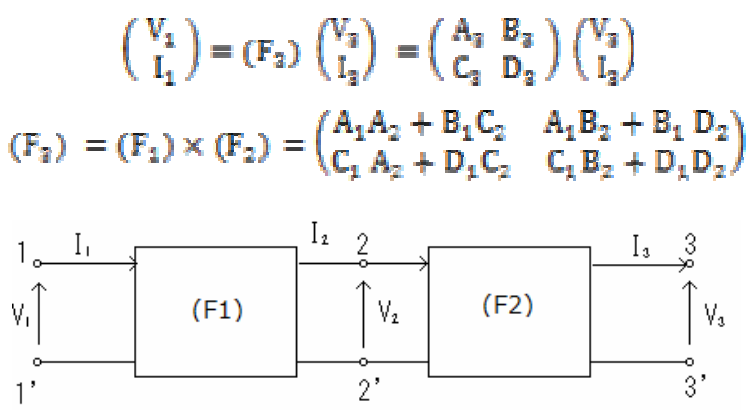

Fig. 2. Cascade connection of two transmission line parameters

Now, Consider input impedance of the transmission line parameters in the two-port network. 2-2' terminal is an output and connects a load impedance, $\mathrm{Z}_{\mathrm{L}}$ as shown in Fig. 3.

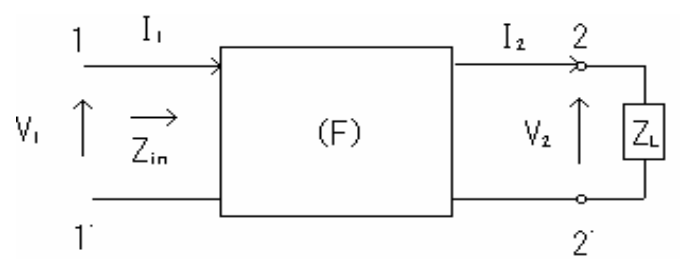

Fig. 3. Connecting a load impedance, $\mathrm{Z}_{\mathrm{L}}$.

Its input impedance, $Z_{\text {in }}$, is given as follows.

$$
\mathrm{Z}_{\text {in }}=\frac{\mathrm{V}_{1}}{\mathrm{I}_{1}}=\frac{\mathrm{AV_{2 }}+\mathrm{BI}_{2}}{\mathrm{CV_{2 }}+\mathrm{DI}_{2}}=\frac{A \mathrm{Z}_{\mathrm{L}}+\mathrm{B}}{\mathrm{CZ_{ \textrm {L } }}+\mathrm{D}}
$$

When 2-2' terminal is opened, or is closed in the tube, $\mathrm{Z}_{\mathrm{L}}$ is infinity or $\mathrm{I}_{2}=0$. Therefore, $\mathrm{Z}_{\text {in }}$ is given as follows.

$$
\mathrm{z}_{\mathrm{in}}=\frac{\mathrm{A}}{\mathrm{C}}[\mathrm{g}
$$

\section{Parameters of Distributed Parameter Circuit for Acoustic Tube}

Characteristic impedance, $\mathrm{W}$, and propagation constant, $\gamma$, for a circuit with loss, are defined as follows. 


$$
\mathrm{W}=\sqrt{G+j w L}, \quad \mathrm{Y}=\sqrt{(\mathrm{R}+j \omega \mathrm{L})\left(\mathrm{G}+\mathrm{H}_{w C}\right.}
$$

$\mathrm{R}, \mathrm{L}, \mathrm{C}$, and $\mathrm{G}$ are a resistance, an inductance, a capacitance and a leakage conductance per unit length of distributed parameter circuit, respectively. $\mathrm{j}$ is a complex unit. When the length of the tube is $l$, transmission line parameters are defined as follows.

$$
\text { (F) }=\left(\begin{array}{cc}
\cosh \gamma l & \text { Wsinhyl } \\
\frac{1}{W} \sinh \gamma l & \cosh \gamma l
\end{array}\right)
$$

Reflection coefficient of distributed parameter circuit, R, is given as follows.

$$
\mathbf{R}=\frac{Z_{a}-Z_{\text {in }}}{Z_{a}+Z_{\text {in }}}
$$

where $Z_{0}$ is an impedance of $\operatorname{air}(=\rho c)$ per $1 \mathrm{~m}^{2}$, and $Z_{\text {in }}$ is an input impedance of the distributed parameter circuit per $1 \mathrm{~m}^{2}$. Then, a normal incident absorption coefficient, a, is calculated as follows.

$$
\mathrm{a}=1-\|\mathbb{R}\|^{2}
$$

Simulations are done by matrix calculation in MATLAB using these equations.

\section{SIMULATION}

\section{Simulation Model and Parameter Constants}

Fig. 4 shows model of perforated panel with a back cavity with/without glass wool, where perforated rate of a panel, its thickness, diameter of its holes, and length of air space are arbitrary. Because the right side of the tube is closed, the right side of the circuit is opened.

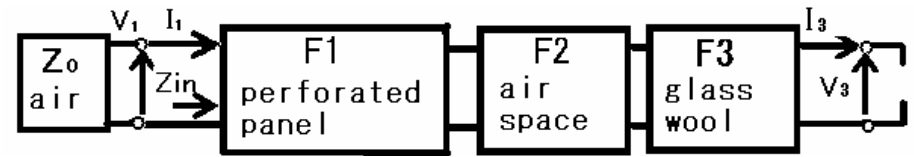

Fig. 4. Model of a perforated panel (F1) with a back cavity (F2) with/without glass wool (F3).

Each hole perforated a panel has loss of viscous friction and thermal conduction, and their parameters are given as follows[3].

$$
\mathrm{L}=\frac{P}{A_{h}}, \mathrm{C}=\frac{A_{h}}{P C^{2}}, \mathrm{R}=\frac{S}{A_{h}^{2}} \sqrt{\frac{\omega \rho \mu}{2}}, \mathrm{G}=\mathrm{S} \frac{\pi-1}{p c^{2}} \sqrt{\frac{\lambda \omega}{2 C_{p} p}}
$$


where $A_{h}$ is the area of the hole, $S$ is the circumference of the hole, $\mu$ is the coefficient of friction, $0.1844 \times 10^{-4}[\mathrm{~Pa} / \mathrm{s}]$, and $\mathrm{C}_{\mathrm{p}}$ is the specific heat at constant pressure, $1005.6\left[\mathrm{~m}^{2} / \mathrm{Ks}^{2}\right], \eta$ is the ration of specific heat at constant pressure to that at constant volume, 1.403 , and $\lambda$ is the coefficient of heat conduction, $23 \times 10^{-3}\left[\mathrm{kgm} / \mathrm{s}^{3} \mathrm{~K}\right]$, and $\omega$ is the angular frequency. For a single hole, it has a characteristic impedance, $\mathrm{W}_{\mathrm{h}}$, and a propagation constant, $\gamma_{h}$, When a panel has $n$ holes, $W=W_{h} / n$, and $\gamma=\gamma_{h}$. Air spaces, or back cavities are assumed as lossless tubes. Their characteristic impedances, $\mathrm{W}_{\mathrm{a}}$, are

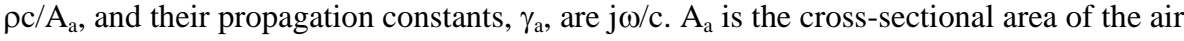
space.

For glass-wool, equations of Miki model is used as follows[5].

$$
\begin{aligned}
& \gamma_{\mathrm{g}}=\frac{\omega}{\mathrm{c}}\left[0.160\left(\frac{f}{\sigma}\right)^{-0.613}+j\left\{1+0.109\left(\frac{f}{\sigma}\right)^{-0.618}\right]\right] \\
& w_{\mathrm{g}}=\operatorname{pc}\left\{1+0.070\left(\frac{f}{\sigma}\right)^{-0.632}-j 0.107\left(\frac{f}{\sigma}\right)^{-0.632}\right\}
\end{aligned}
$$

where $\sigma$ is flow resistance of the glass-wool, $\sigma$ is $10000\left[\mathrm{~Pa} \mathrm{~s} / \mathrm{m}^{2}\right]$ for $96 \mathrm{~kg} / \mathrm{m}^{3}$ glass wool, and $\mathrm{f}$ is frequency[Hz].

\section{SIMULATION RESUTLS AND DISCUSSIONS}

\section{Simulation for Perforated Panel with Back Cavity, or MPP}

Fig. 5 shows measurement[6] and simulation of normal incident sound absorption coefficient for $1 \mathrm{~mm}$ thickness of the panel, $1 \mathrm{~mm}$ diameter of holes, $1 \%$ perforation rate, and $50 \mathrm{~mm}$ length of the back cavity.

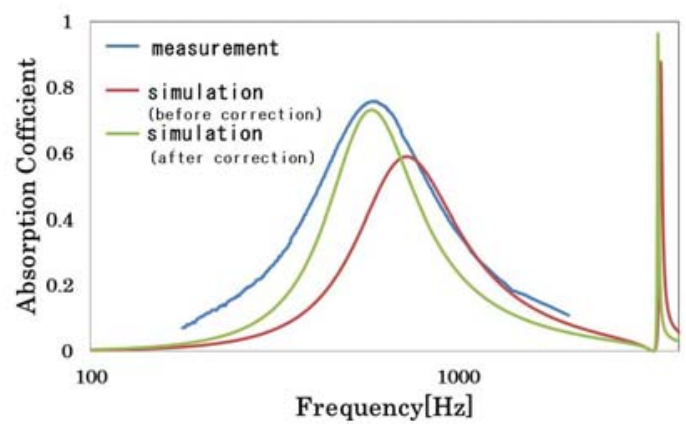

Fig. 5. Measurement and simulation (before/after correction) of normal incident sound absorption coefficient for $1 \mathrm{~mm}$ thickness of the panel, $1 \mathrm{~mm}$ diameter of holes, and $1 \%$ perforation rate.

Red curve is shown for $1 \mathrm{~mm}$ thickness of the panel, but is not good agreement with the measurement results. Therefore, open-end correction is considered[7]. When the radius of the holes is defined as $\mathrm{r}$, open-end correction is $0.61 \times \mathrm{r}$. Since open-end corrections are 
done in the both sides of the holes, the effective thickness of the panel is $1.61 \mathrm{~mm}$. Bright green curve is open-end corrected, and it is good agreement with blue curve, or the measurement curve. Afterward, for simulation open-end corrections are done, and the effective thickness of the panel is given as follows:

$$
\text { tp }+0.61 \times d,
$$

where tp is the thickness of the panel, and $d$ is the diameter of the holes.

\section{Simulation of Absorption Coefficients for Various Perforated Rates}

Fig. 6 shows normal incident absorption coefficients for various perforated rates of the panels by simulation. This simulation is done as composition of Fig. 4 except F3. The maximum values of absorption coefficients are almost 1 in the case of $0.25 \%$ perforated rate, and for $1 \%$ perforated rate they are about 0.7 . Fig. 7 shows measurement by the transfer function method for $0.1 \%$ to $1.25 \%$ perforated rates. The maximum values of absorption coefficients are good agreements with those of simulation, but their frequencies are 10 to $15 \%$ lower than those of simulation.
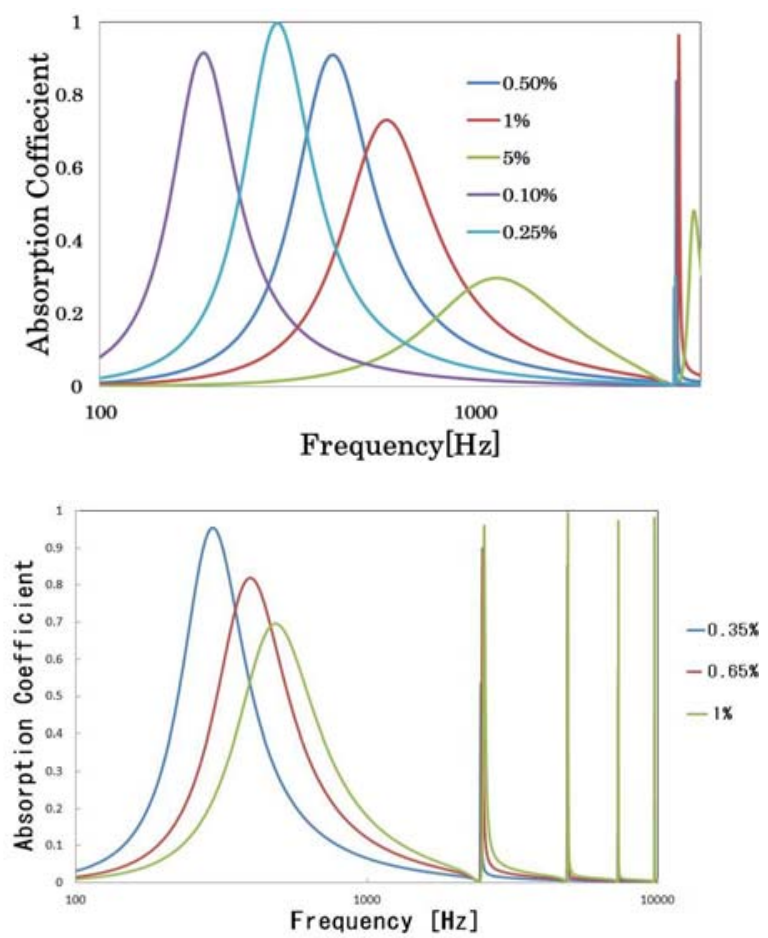

Fig. 6. Normal incident absorption coefficients for various perforated rates of the panels by simulation. The diameter of the holes is $1 \mathrm{~mm}$. The back cavities are $50 \mathrm{~mm}$ long (top) and 70 $\mathrm{mm}$ long (bottom). The thickness of the panel is $1 \mathrm{~mm}$. 
Simulation Of Normal Incidence Sound Absorption Coefficients Of Perforated Panels

\section{Simulation of Absorption Coefficients for Various Thickness of Panels and Diameter} of Holes

Fig 8 (a) shows absorption coefficients for $0.5 \mathrm{~mm}$ to $10 \mathrm{~mm}$ thickness of the panels, $1 \%$ perforated rate, $1 \mathrm{~mm}$ diameter of the holes and $50 \mathrm{~mm}$ length of the back cavity by simulation. When the panel is $8 \mathrm{~mm}$ thick, the maximum value of absorption coefficients is the highest and almost 1 . Fig 8 (b) shows those for 0.5 to $5 \mathrm{~mm}$ diameter of holes, $1 \%$ perforated rate, $1 \mathrm{~mm}$ thickness of the panels by simulation. The larger the diameter of the holes is, the lower the absorption coefficients are. When the diameters of the holes are 2 and $5 \mathrm{~mm}$, the maximum values of absorption coefficients are lower than those of measurements.

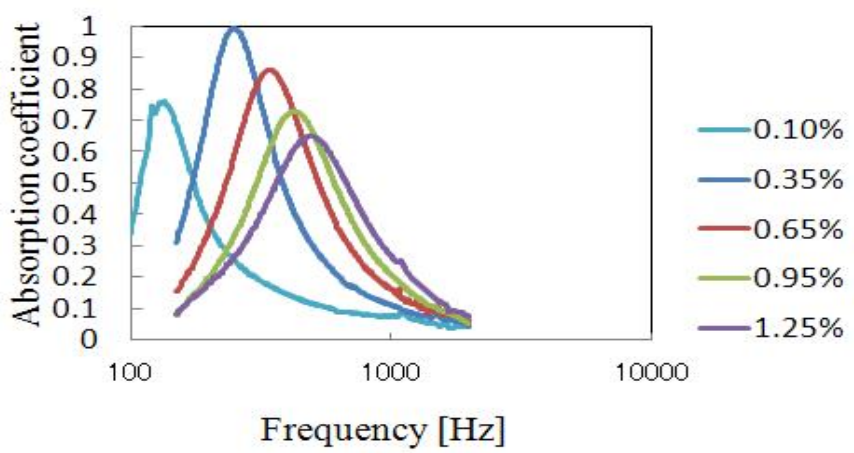

Fig. 7. Normal incident absorption coefficients for $0.1 \%$ to $1.25 \%$ perforated rates of the panels by measurement (transfer function method). The back cavity is $70 \mathrm{~mm}$ long. The diameter of holes is $1 \mathrm{~mm}$. The thickness of the panel is $1 \mathrm{~mm}$.

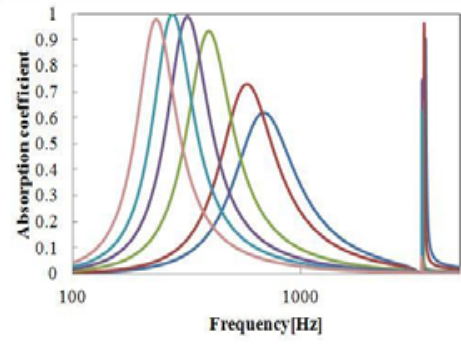

(a)

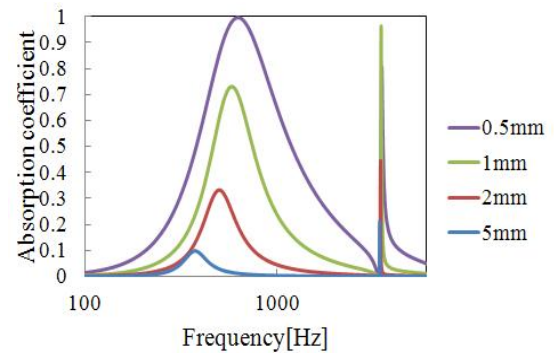

(b)

Fig. 8. Normal incident absorption coefficients (a) for 0.5 to $10 \mathrm{~mm}$ thickness of the panels and $1 \mathrm{~mm}$ diameter of the holes by simulation and (b) for 0.5 to $5 \mathrm{~mm}$ diameter of the holes and $1 \mathrm{~mm}$ thickness of the panels (right). The perforated rates are $1 \%$ and the back cavities are $50 \mathrm{~mm}$. 

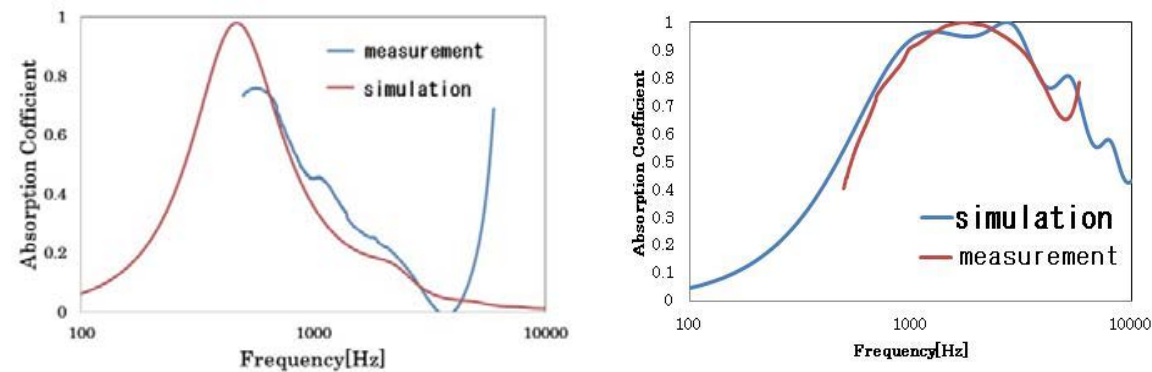

Fig. 9. Normal incident absorption coefficients of measurement by transfer function method and simulation for $1 \mathrm{~mm}$ thickness of $1 \%$ (left) and 8.2\%(right) perforated panel, $1 \mathrm{~mm}$ diameter of holes of the perforated panel, $10 \mathrm{~mm}$ air space, and $50 \mathrm{~mm}$ thickness of $96 \mathrm{~kg} / \mathrm{m}^{3}$ glass-wool.

\section{Simulation of Absorption Coefficients for Back cavity with Glass-wool}

Glass-wool absorption materials are inserted between the perforated panel and the right end of the tube. The thickness of $1 \%$ and $8.2 \%$ perforated panels is $1 \mathrm{~mm}$, the air space is $1 \mathrm{~cm}$, and $96 \mathrm{~kg} / \mathrm{m}^{3}$ glass-wool is $5 \mathrm{~cm}$ thick. The diameter of holes of the perforated panel is $1 \mathrm{~mm}$. These simulations are done as composition of Fig 4. The measurement is done from $500 \mathrm{~Hz}$ to $5.6 \mathrm{kHz}$ by the transfer function method [6]. Simulations are almost good agreement with their corresponding measurements as shown in Fig 9.

\section{Conclusion}

This paper described simulation of normal incident sound absorption coefficients for perforated panels by transmission line parameters in a two-port network of distributed parameter circuit. It is shown that simulations are almost good agreements with measurements by transfer function method for various conditions such as perforated rates of the panels, diameters of the holes, and back cavity with/without glass wool. In the some cases, measurements are not done. So, measurements will be done, and these results and the corresponding simulations will be weighed.

\section{References}

[1] D. Y. Maa, ”Theory and design of microperforated panel sound-absorbing construction,” Sci. Sin., 18,pp.55-71(1975)

[2] K. Sakagami, S. Kobatake, K.Kano, M. Morimoto, and M.Yairi, "Sound absorption characteristics of a single microperforated panel absorber backed by a porous absorbent layer," Acoustics Australia, 39, 3, pp.95-100 (2011)

[3] L. R. Rabiner and R. W. Schafer, "Digital Processing of Speech Signals,” Prentice-Hall (1978)

[4] R. Sato, “Transmission circuit,” (in Japanese) Corona publishing co. (1972)

[5] Y. Miki, “Acoustical properties of porous materials-Modifications of Delany-Bazley models-“ J.Acoust.Soc.Jpn(E) 11, 1 (1990)

[6] T. Nakai, F. Kawakami, K. Wada and T. Sano, "Acoustical Characteristics Newly Developed Perforated Plates,” Proceeding of $20^{\text {th }}$ of ICA, 757 (2010)

[7] H. Levine and J. Schwinger , "On the Radiation of Sound from an Unflanged Circular Pipe,” Physical review, 73, 4 , P383-406 (1947) 